\title{
Effects of trilinear symmetry breaking on the Potts-model transition of uniaxially stressed $\mathrm{SrTiO}_{3}$
}

\author{
José F. Fontanari* and W. K. Theumann \\ Instituto de Fisica, Universidade Federal do Rio Grande do Sul, 90049 Porto Alegre, Rio Grande do Sul, Brazil
}

(Received 7 February 1985; revised manuscript received 24 September 1985)

\begin{abstract}
A Landau analysis shows that a break of trilinear symmetry in the continuous three-state Potts model with linear and quadratic symmetry breaking is relevant for the trigonal-to-pseudotetragonal phase transition in uniaxially stressed $\mathrm{SrTiO}_{3}$ along $[1+\delta 1+\delta 1-2 \delta]$, and that the effects of quartic symmetry breaking are vanishingly small. The shift of the tricritical stress parameter $\delta_{t}$ is large enough to mask fluctuation corrections to $\delta_{t} / \delta_{c}$ in $d=4-\epsilon$ dimensions, and a number of quantitative results for the phase diagrams are obtained.
\end{abstract}

Attention has been drawn recently to the study of the effects of symmetry-breaking perturbations on the phase transition of the continuous version of the $p$-state Potts model, motivated by physical realizations of the model. ${ }^{1-6}$ Of particular interest is the three-state model which has been pointed out to describe, among other things, the magnetic phase transition in cubic ferromagnets with easy axes along the cubic axes in a [111] diagonal magnetic field, ${ }^{7}$ and the first-order trigonal-topseudotetragonal structural phase transition in [111]stressed perovskites like $\mathrm{SrTiO}_{3} .{ }^{8}$ Blankschtein and Aharony (BA) suggested further than an off-diagonal stress along $[1+\delta, 1+\delta, 1-2 \delta]$ applied to $\mathrm{SrTiO}_{3}$ should yield to a trigonal-to-pseudotetragonal phase transition described by the three-state Potts model with linear and quadratic symmetry breaking. ${ }^{2(b)}$ The effect of these symmetry-breaking terms is to change the first-order transition for this model into a second-order transition at a tricritical point or the first-order transition may disappear at a critical point. Also, universal ratios of critical and tricritical parameters (reduced temperature, "magnetic field," and order parameter) were proposed on the basis of scaling arguments and confirmed by renormalizationgroup ( $R G)$ calculations, to order $\epsilon$, in $d=4-\epsilon$ dimensions. $^{2(b)}$

It has been pointed out that quadratic symmetry breaking yields a break of trilinear symmetry in the continuous version of the Potts model, ${ }^{4,6,9}$ but a physical realization has not been discussed before. In the present paper we provide mean-field estimates of the effects of trilinear symmetry breaking (TSB) on the Potts-model transition of uniaxially stressed $\mathrm{SrTiO}_{3}$ with the main purpose of finding out if fluctuation corrections should take into account the full trilinear coupling in a RG calculation in $d=6-\epsilon$ dimensions, and eventually to stimulate further experimental and theoretical work. We also estimate the effects of quartic symmetry breaking (QSB), which should be considered together with TSB in the case of $\mathrm{SrTiO}_{3}$.

Our main results are the following: (a) the shift of the tricritical point due to TSB is large enough to mask the fluctuation corrections calculated with the RG in $d=4-\epsilon$ dimensions to the ratio $\delta_{t} / \delta_{c}$ of the tricritical and critical stress parameters, $\delta_{t}$ and $\delta_{c}$. (b) TSB does not alter the qualitative picture of the phase diagrams of BA I. (c) Quantitative estimates of the effects of TSB on the phase diagrams are obtained in terms of appropriate dimensionless parameters that contain the experimental variables $p$, $T$, and $\delta$. (d) It is shown that QSB provides negligible corrections to the results with TSB.

We follow the Landau analysis for perovskites of BA II and take the free-energy functional for uniaxial stress $\sigma_{i j}=-p \alpha_{i} \alpha_{j}$ in the direction of the unit vector with components $\alpha_{i}$ as

$$
\begin{aligned}
F= & \frac{1}{2} K|\mathbf{Q}|^{2}+A|\mathbf{Q}|^{4} \\
& +A_{n} \sum_{i<j} Q_{i}^{2} Q_{j}^{2}+b_{e} p \sum_{i}\left(3 \alpha_{i}^{2}-1\right) Q_{i}^{2} \\
& +b_{t} p \sum_{i<j} \alpha_{i} \alpha_{j} Q_{i} Q_{j},
\end{aligned}
$$

where $b_{e}$ and $b_{t}$ are coefficients that couple elastic to order-parameter degrees of freedom in the cubic system with three-component order parameter $Q$ that represents the high-temperature phase of $\mathrm{SrTiO}_{3}$ beyond the secondorder pseudocubic-to-trigonal phase transition. ${ }^{8}$ Experimental values for all five constants are available in the literature. ${ }^{10}$ With the stress along $[1+\delta, 1+\delta, 1-2 \delta]$, and rotating the order parameter to bring one component along [111], as $s_{1}=\left(Q_{1}+Q_{2}+Q_{3}\right) / \sqrt{3}, s_{2}=\left(Q_{1}-Q_{2}\right) /$ $\sqrt{2}$, and $s_{3}=\left(Q_{1}+Q_{2}-2 Q_{3}\right) / \sqrt{6}$, Eq. (1) becomes

$$
\begin{aligned}
F= & \frac{1}{2} r_{1} s_{1}^{2}+\frac{1}{2} r_{2} s_{2}^{2}+\frac{1}{2} r_{3} s_{3}^{2}+u_{0}|\mathbf{s}|^{4} \\
+ & v_{0}\left[\frac{1}{3} s_{1}^{4}+2 s_{1}^{2}\left(s_{2}^{2}+s_{3}^{2}\right)+\frac{1}{2}\left(s_{2}^{2}+s_{3}^{2}\right)^{2}\right. \\
& \left.-(2 \sqrt{2} / 3)\left(s_{3}^{3}-3 s_{3} s_{2}^{2}\right) s_{1}\right]+g_{13} s_{1} s_{3},
\end{aligned}
$$

in which $u_{0}=A+A_{n} / 2, v_{0}=-A_{n} / 2$, and

$$
\begin{aligned}
& r_{1}=K+\frac{2}{3} b_{t} p+O\left(\delta^{2}\right), \\
& r_{2}=K-\frac{1}{3} b_{t} p+\frac{2}{3}\left(6 b_{e}-b_{t}\right) p \delta+O\left(\delta^{2}\right), \\
& r_{3}=K-\frac{1}{3} b_{t} p-\frac{2}{3}\left(6 b_{e}-b_{t}\right) p \delta+O\left(\delta^{2}\right), \\
& g_{13}=(\sqrt{2} / 3)\left(12 b_{e}+b_{t}\right) p \delta+O\left(\delta^{2}\right),
\end{aligned}
$$

where $b_{e}>0, b_{t}<0$, but $6 b_{e}-b_{t}>0$, for $\mathrm{SrTiO}_{3}$. 
Since $r_{1}<\left(r_{2}, r_{3}\right)$, the order-parameter component $s_{1}$ orders first as the temperature is lowered from the pseudocubic phase at constant $p$. The value of $s_{1}$ that minimizes $F$ gives the trigonal order parameter

$$
s_{1}=M=\left(\frac{-r_{1}}{4\left(u_{0}+v_{0} / 3\right)}\right)^{1 / 2}, s_{2}=s_{3}=0 .
$$

As the temperature is lowered further either $s_{2}$ or $s_{3}$ (eventually both) will order next, depending on the sign of $\delta$ for which the coefficients $\widetilde{r}_{2}=r_{2}+4\left(u_{0}+v_{0}\right) M^{2}$ and $\widetilde{r}_{3}=r_{3}+4\left(u_{0}+v_{0}\right) M^{2}$ of the terms in $s_{2}^{2}$ and $s_{3}^{2}$ vanish. These new coefficients include the contributions due to the shift of $s_{1}$ to $s_{1}=M+\widetilde{s}_{1}$ by the small secondary order parameter $\tilde{s}_{1}$, given by $\tilde{s}_{1}=-\left[\tilde{w}_{1}\left(s_{2}^{2}+s_{3}^{2}\right)+g_{13} s_{3}\right] / \tilde{r}_{1}$, where $\widetilde{r}_{1}=r_{1}+4\left(3 u_{0}+v_{0}\right) M^{2}$ and $\widetilde{w}_{1}=4\left(u_{0}+v_{0}\right) M$. Eliminating $\tilde{s}_{1}$, as in BA II, we obtain to leading order in $\delta$

$$
\begin{aligned}
F(\mathrm{~s})= & \frac{1}{2} r\left(s_{2}^{2}+s_{3}^{2}\right)-\frac{1}{2} g\left(s_{3}^{2}-s_{2}^{2}\right)+u\left(s_{3}^{3}-3 \frac{v}{u} s_{2}^{2} s_{3}\right) \\
& +u_{4}\left(s_{2}^{2}+s_{3}^{2}\right)^{2}-\hat{u}\left(s_{3}^{4}-3 s_{2}^{2} s_{3}^{2}\right)-h_{3} s_{3},
\end{aligned}
$$

where $r=\tilde{r}_{2}=\widetilde{r}_{3}$ at $\delta=0$,

$$
g=\frac{1}{2}\left(\widetilde{r}_{3}-\widetilde{r}_{2}\right)=\frac{2}{3}\left(6 b_{e}-b_{t}\right) p \delta+O\left(\delta^{2}\right),
$$

with the "external field" $h_{3}=-g_{13} M$, in which $g_{13}$ is given by Eq. (6), while $u_{4}=u_{0}+\frac{1}{2} v_{0}-\widetilde{w}_{1}^{2} / 2 \widetilde{r}_{1}$, $\hat{u}=-(2 \sqrt{2} / 3) v_{0} g_{13} / \widetilde{r}_{1}$, and

$$
\begin{aligned}
& u=w\left(1+3 \frac{\left(u_{0}+v_{0}\right) g_{13}}{4 \sqrt{2} v_{0} M^{2}\left(u_{0}+v_{0} / 3\right)}\right), \\
& v=w\left(1-\frac{\left(u_{0}+v_{0}\right) g_{13}}{4 \sqrt{2} v_{0} M^{2}\left(u_{0}+v_{0} / 3\right)}\right),
\end{aligned}
$$

in which $w=-(2 \sqrt{2} / 3) v_{0} M$. Equation (8) is then recognized as the free energy for the three-state Potts model with linear, quadratic, and TSB terms, ${ }^{6,11}$ with QSB. Note from Eqs. (3), (6), and (7) that the parameters $p, \delta$, and $K$ that can be varied experimentally appear only in the dimensionless form $g_{13} / M^{2} v_{0}$. For the purpose of constructing phase diagrams that can be both useful for experiments and for comparison with those of BA I, it is convenient to define dimensionless parameters

$$
\begin{aligned}
& R=\left(4 u_{4} / 9 w^{2}\right) r, \\
& H=\left(16 u_{4}^{2} / 27 w^{3}\right) h_{3},
\end{aligned}
$$

and

$$
G=\left(4 u_{4} / 9 w^{2}\right) g,
$$

in which $w=u(1+3 q) / 4$, where $q \equiv v / u$. With this choice of parameters,

$$
G=\frac{u_{4}}{3 v_{0}^{2}}\left(6 b_{e}-b_{t}\right) \frac{p \delta}{M^{2}},
$$

and noting that $g_{13} \propto p \delta$, Eq. (6), $u / w$, and $v / w$ will only depend on $G$ (besides a constant) for $\mathrm{SrTiO}_{3}$. Although when $\delta \neq 0$ there is no longer a "true" trigonal phase in which $s_{1}=M \neq 0$ but $s_{2}=s_{3}=0$, because the field term $h_{3} s_{3}$ will induce a secondary ordering of $s_{3}$ along $h_{3}$, we follow BA II in assuming that, at least to lowest order in $\delta$, our results should give the correct description of the trigonal-to-pseudotetragonal phase transition in $\mathrm{SrTiO}_{3}$.

To determine the shift of the critical and tricritical point due to TSB we constructed the phase diagrams that follow from $\mathrm{Eq}$. (8) in the standard way. ${ }^{1}$ Some general results are first obtained for the Potts model which we later apply to $\mathrm{SrTiO}_{3}$. The results for the former are the following: first we find that the qualitative picture of the phase diagrams is the same as that of BA I, without TSB. There are three equilibrium phases: in phase $I s_{3} \neq 0$, $s_{2}=0$, but $s_{3} \rightarrow 0$ in the limit $h_{3} \rightarrow 0$. This is the disordered phase of the model and it corresponds to the trigonal phase for $\mathrm{SrTiO}_{3}$. Next, in phase II $s_{3} \neq 0, s_{2}=0$, but $s_{3} \nrightarrow 0$ as $h_{3} \rightarrow 0$. In phase III $s_{3} \neq 0$ and $s_{2} \neq 0$. These two are ordered phases separated from I by a first-order transition ending at a critical point (in the case of phase II) or changing into a continuous transition in which I and III are in equilibrium beyond a tricritical point. The tricritical point eventually disappears at a critical endpoint, and a typical phase diagram is shown in Fig. 1. The quantitative effect of TSB is to lower the first-order transition line II-III in a way that shortens the line I-II. At the same time the tricritical point is raised. None of the qualitative features are changed by QSB and the quantitative effect is a small perturbation due to the smallness of $q_{4} \equiv \hat{u} / u_{4} \cong 2.6 \times 10^{-2} G$ for $\mathrm{SrTiO}_{3}$.

Indeed, the critical point is given by

$R_{c}=G+\frac{16}{3 \hat{q}^{2}\left(1-q_{4}\right)}, \quad H_{c}=-\frac{64}{27 \hat{q}^{3}\left(1-q_{4}\right)^{2}}$

in which $\hat{q} \equiv 1+3 q$ and the value of the order parameter along the critical line remains constant at $s_{3}=M_{c}(G)=-u / 4 u_{4}\left(1-q_{4}\right)$. Second, the line of firstorder transition between phase I and II is given by

$R_{\mathrm{I}-\mathrm{II}}=\frac{1}{9}\left(\frac{32}{\hat{q}^{2}\left(1-q_{4}\right)}-27 \hat{q}\left(1-q_{4}\right) H / 4+9 G\right), H \geq H_{c}$.

The tricritical point is located at

$$
\begin{aligned}
R_{t}(G, q)=256 \frac{m}{\hat{q}^{2}}\{ & \left(\frac{1}{2} q-m\right)-\frac{1}{256} \frac{\hat{q}^{2}}{m} G \\
& \left.+\frac{3}{2} q_{4} \frac{1}{\hat{q}}[(q-4 m)(7 m-q)-m \hat{q}]\right\},
\end{aligned}
$$

$$
\begin{aligned}
H_{t}(G, q)=1024 \frac{m}{\hat{q}^{3}}\left\{\frac{1}{2} q^{2}-m(1+4 q)\right. & \\
+\frac{1}{2} q_{4} \frac{1}{\hat{q}}[ & 3 q(q-4 m)(7 m-q) \\
& \left.\left.-20 m^{2} \hat{q}\right]\right\},
\end{aligned}
$$

in which $m \equiv\left(16 q^{2}+\hat{q}^{2} G\right) / 64 \hat{q}$ and the value of $s_{3}$ along 
the tricritical line is given by

$$
s_{3}(G, q)=3 \frac{u}{u_{4}} m\left[1+3 \frac{q_{4}}{\hat{q}}(7 m-q)\right] .
$$

For $\mathrm{SrTiO}_{3}$ we find that the tricritical point becomes a critical endpoint when $G \geq 0.563$, a limiting value for which $R_{t} \cong 0.252$ and $H_{t} \cong 0.084$. On the other hand, the critical point disappears when $G \leq-0.200$, for which $R_{c} \cong 0.226$ and $H_{c} \cong-0.053$, with an error only in the last figures due to neglect of QSB.
Using the relationships for $R_{c}$ and $H_{c}$ in terms of the physical parameters of the model given by Eq. (1), we find for the critical point,

$$
\begin{aligned}
& p_{c} \delta_{c}=\frac{A_{n}^{3} M_{c}^{2}}{72 u_{4}^{2}\left(12 b_{e}+b_{t}\right)}\left[1+z\left[1-\frac{1}{27} \frac{A_{n}^{2}}{u_{4} A}\right)\right]^{-1}, \\
& p_{c}=\frac{3 K(1-\hat{y})}{b_{t}(1+2 \hat{y})}
\end{aligned}
$$

with

$\hat{y}=\left[4 A-\left(A_{n}^{2} / 6 u_{4}\right)-\left[A_{n}^{3}\left(6 b_{e}-b_{t}\right) / 108 u_{4}^{2}\left(12 b_{e}+b_{t}\right)(1+z)\right]+A_{n}^{2} z\left[1-\frac{1}{36} \frac{A_{n}^{2}}{u_{4} A}\right] / 9 u_{4}(1+z)\right] / 4\left(A+A_{n} / 3\right)$

in which

$$
z=\frac{A_{n}^{2} A}{48 u_{4}^{2}\left(A+A_{n} / 3\right)}
$$

is the term that corrects for TSB and the terms in $A_{n}^{2} / u_{4} A$ are the contributions from QSB, while

$$
\begin{aligned}
& u_{4}=A+\left(A_{n} / 4\right)-A^{2} /\left(A+A_{n} / 3\right), \\
& M_{c}^{2}=-\left(K+\frac{2}{3} b_{t} p_{c}\right) /\left[4\left(A+A_{n} / 3\right)\right] .
\end{aligned}
$$

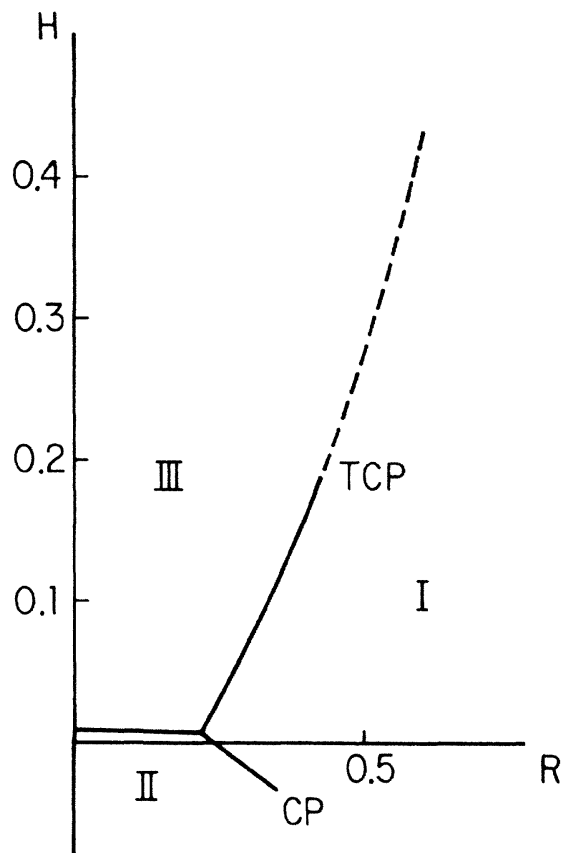

FIG. 1. Phase diagram for the three-state Potts model with linear, quadratic, and TSB in terms of dimensionless $H, R$, and $G$, for fixed $G=0.0298$ corresponding to $u=0.9798$ and $v=1.0067$ (Ref. 11, with $\hat{g}=0.217 G$ ). First-order transitions ending at a critical point (CP) or at a tricritical point (TCP) represented as solid lines and the broken line indicates a second-order transition. Since $G_{c} \cong 0.0298, \mathrm{CP}$ also lies on a line of critical points in $(p, T)$ space for $\mathrm{SrTiO}_{3}$ if $\delta$ is fixed at $\cong \delta_{c}=0.0077$.
With the measured parameters for $\mathrm{SrTiO}_{3}$ (in cgs units) at $4.2 \mathrm{~K}, K=-3.08 \times 10^{25}, \quad A=1.58 \times 10^{43}, \quad A_{n}=6.19$ $\times 10^{42}, b_{e}=7.34 \times 10^{14}$, and $b_{t}=-1.98 \times 10^{15}$ we find

$p_{c}=25.15(25.38) \mathrm{kg} \mathrm{mm}^{-2}, \delta_{c}=0.0077(0.0081)$

$\alpha_{3 c}=0.5685(0.568), M_{c}=6.904(6.907) \times 10^{-10} \mathrm{~cm}$

and for the dimensionless parameter $G$ we obtain $G_{c}=0.0298$, where in parenthesis are our values without TSB and QSB. Neglect of QSB leaves these results unchanged, except for the last figure in $\alpha_{3 c}$. In view of the relatively large uncertainties in the determination of the parameters by present experiments, ${ }^{10}$ the predicted shift in $\delta_{c}, \Delta \delta_{c}=4 \times 10^{-4}$ is too small to be detected. The effects on the tricritical point, however, are quite larger.

Equations (15) yield for the tricritical point

$$
\begin{aligned}
& p_{t} \delta_{t}= \hat{y}_{t} M_{t}^{2}, \\
& \hat{y}_{t}=\left(9 A_{n}^{3} / 128 u_{4}^{2}\right)\left\{\left[A_{n}\left(6 b_{e}-b_{t}\right) / 16 u_{4}\right]-\left(12 b_{e}+b_{t}\right)\right. \\
&\left.\times\left[1+\frac{33 z}{16}\left[1-\frac{101}{2112} \frac{A_{n}^{2}}{u_{4} A}\right]\right]\right\}^{-1}, \\
& p_{t}=3 K\left(1-\hat{y}_{2}\right) / b_{t}\left(1+2 \hat{y}_{2}\right), \\
& \hat{y}_{2}=\left\{4 A-\frac{7 A_{n}^{2}}{32 u_{4}}+\frac{5}{12}\left(6 b_{e}-b_{t}\right) \hat{y}_{t}-\frac{5}{48 u_{4}} \frac{A_{n} A}{A+A_{n} / 3}\right. \\
&\left.\quad \times\left(12 b_{e}+b_{t}\right)\left[1-\frac{43}{1280} \frac{A_{n}^{2}}{u_{4} A}\right] \hat{y}_{t}\right\} /\left[4\left(A+A_{n} / 3\right)\right],
\end{aligned}
$$

where the correction due to TSB is in the last two terms of $\hat{y}_{2}$, and QSB is in the term $43 A_{n}^{2} / 1280 u_{4} A$, $M_{t}^{2}=-\left(K+\frac{2}{3} b_{t} p_{t}\right) /\left[4\left(A+A_{n} / 3\right)\right]$. With the same measured parameters we find, with no significant change due to QSB,

$p_{t}=27.39(28.27) \mathrm{kg} \mathrm{mm}^{-2}, \quad \delta_{t}=-0.0378(-0.0419)$

$\alpha_{3 t}=0.621(0.626), M_{t}=6.933(6.944) \times 10^{-10} \mathrm{~cm}$

$G_{t} \cong-0.158$ 
It can easily be seen from Eqs. (17)-(19), (24), and (25) that $\delta_{c}$ and $\delta_{t}$ remain the same with varying $K$ and $p$.

The result obtained here for $\delta_{t} / \delta_{c} \cong-4.901$ with, or $\cong-4.896$ without $\mathrm{QSB}$ should now be compared with the mean-field prediction $\delta_{t} / \delta_{c} \cong-5.18$ of BA II, without TSB and QSB. Although the difference is presumably too small to be detected experimentally, at present, it is interesting to note that it is much larger than the effect of fluctuation corrections in $d=4-\epsilon$ dimensions, calculated in BA II, where

$$
\delta_{t} / \delta_{c} \cong-5(81 / 80)^{\Delta / \phi-3 / 2},
$$

in which $\Delta / \phi-\frac{3}{2} \cong-\epsilon / 10+O\left(\epsilon^{2}\right)$. This yields $\delta_{t} / \delta_{c} \cong-4.9988$ in three dimensions, which should be compared with the result $\delta_{t} / \delta_{c} \cong-5$, when $\epsilon=0$ for the fluctuation-free theory of Ref. 2(b), based on the XY model. An appreciable effect on $\delta_{t} / \delta_{c}$ implies also one on the ratio of the fields $h_{3 t} / h_{3 c}$. One of our main results, that TSB completely masks the fluctuations corrections in $d=4-\epsilon$ dimensions, suggests that it should be interesting to study the effect of fluctuation corrections which take fully into account the trilinear coupling of the model by means of RG calculations in $d=6-\epsilon$ dimensions. Some progress in this direction will be reported elsewhere. ${ }^{12}$ Our other main result, that the Potts-model transition of uniaxially stressed $\mathrm{SrTiO}_{3}$ is a physical realization of TSB, due to the negligible effect of QSB, may be a further motivation to consider such RG calculations. The results of the present work are expected to apply along the Potts-model transition line in uniaxially stressed $\mathrm{SrTiO}_{3}$, except in the immediate neighborhood of the multicritical point where the continuous trigonal to pseudocubic transition starts, for which the trilinear coupling should be vanishing small.

We acknowledge stimulating comments by Dr. Daniel Blankschtein on an earlier version of this paper and for raising the possible relevance of quartic symmetry breaking. This work was supported in part by Conselho Nacional de Desenvolvimento Científico e Tecnológico (CNPq) and Financiadora de Estudos e Projetos (FINEP), Brasil. J.F.F. also acknowledges support from PróReitoria de Pesquisa e Pós-Graduação_(PROPESP)Universidade Federal do Rio Grande do Sul and CNPq.
*Present address: Instituto de Física e Química de São Carlos, Universidade de São Paulo, 13560 São Carlos, São Paulo, Brazil.

${ }^{1}$ D. Blankschtein and A. Aharony, J. Phys. C 13, 4635 (1980), referred to in the text as BA I.

${ }^{2}$ (a) D. Blankschtein and A. Aharony, Phys. Rev. B 22, 5549 (1980); (b) J. Phys. C 14, 1919 (1981), referred to in the text as BA II.

${ }^{3}$ M. J. Stephen and G. S. Grest, Phys. Rev. Lett. 38, 567 (1977).

${ }^{4}$ W. K. Theumann and Alba Theumann, Phys. Rev. B 24, 6766 (1981); Alba Theumann and W. K. Theumann, ibid. 26, 3856 (1982).

${ }^{5}$ W. K. Theumann, Phys. Rev. B 27, 6941 (1983).

6W. K. Theumann, Phys. Rev. B 28, 6519 (1983).

${ }^{7}$ D. Mukamel, M. E. Fisher, and E. Domany, Phys. Rev. Lett.
37,565 (1976).

${ }^{8}$ A. Aharony, K. A. Müller, and W. Berlinger, Phys. Rev. Lett. 38, 33 (1977).

${ }^{9}$ Marcia C. Barbosa, Master thesis, Universidade Federal do Rio Grande do Sul, 1984 (unpublished).

${ }^{10}$ K. A. Müller, W. Berlinger, and J. C. Slonczewski, Phys. Rev. Lett. 25, 734 (1970); J. C. Slonczewski, Phys. Rev. B 2, 4646 (1970).

${ }^{11}$ Equations (10) and (11) are in agreement with a general result for the $p=(n+1)$-state continuous Potts model, with dimensionless quadratic-symmetry breaking parameter $\hat{g}$, that $u \cong w[1+3(n-1) \hat{g}]$ and $v \cong w[1+(n-3) \hat{g}]$, for small $\hat{g}$, Ref. 9.

${ }^{12}$ Marcia C. Barbosa, M. A. Gusmão, and W. K. Theumann (unpublished). 